Asian Journal of Information Technology 10 (2): 96-100, 2011

ISSN: $1682-3915$

(C) Medwell Journals, 2011

\title{
Some Advances and Prospects in Nanotechnology: A Review
}

\author{
J.C. Osuwa and P.C. Anusionwu \\ Department of Physics, Micheal Okpara University of Agriculture, \\ P.M.B. 7267, Umudike, Abia State, Nigeria
}

\begin{abstract}
This study surveys controlled manipulation and manufacture of materials and devices at extremely small scale termed nanotechnology. The profound impact of the applications of nanoscale materials ranging from 1-100 nm, on information technology, electronic and optoelectronic devices, medicine, environment and human life are highlighted. The attainment of these unique capabilities by nanomaterials due to their novel characteristics and properties such as preponderance of large surface to volume ratios and quantum effects as manifested in quantum dots, quantum wells, atomic and magnetic clusters and leading to high efficiency lasers with specific wavelengths, materials of exceptional strengths and other novel effects are examined. A holistic discourse of Nanotechnology encompassing its conceptual, developmental and practical and application stages are also presented. The benefits, challenges and implications of this emerging revolution of miniaturized technology are evidently intriguing.
\end{abstract}

Key words: Quantum dots, quantum wells, atomic clusters, microscopes, intrigning, Nigeria

\section{INTRODUCTION}

Nanotechnology is simply the manipulation and manufacture of materials and devices at extremely small scales ranging from 1-100 nm. Comparatively, $1 \mathrm{~nm}$ which is 1 billionth of a meter is about 50,000 times smaller than the diameter of a human hair while the diameter of the smallest naturally occurring atom, the hydrogen atom is only $0.1 \mathrm{~nm}$. The proteins that carry out the internal operations of the cell are just $3-20 \mathrm{~nm}$ in size and so have nanoscale dimensions. Viruses that attack human cells are about 10-200 nm and the molecules in the drugs used to fight viruses are $<5 \mathrm{~nm}$ in size. The nanoscale is unique because many mechanisms of the biological and physical world operate at the level of nanoscales from 0.1-100 nm. At these dimensions, materials exhibit distinctive physical and chemical properties. Thus, many envisaged novel effects at the nanoscale are expected to bring about tremendous technological breakthroughs.

Indeed, a number of important break throughs have already occurred in nanotechnology. Some examples include catalytic converters in automobiles that help remove air pollutants, devices in computers that help read from and write to hard disk, zinc oxide nanocrystals used to create invisible sunscreens and added in cosmetics that block harmful ultraviolet radiations from the sun, special coatings for sports clothes that help improve the gear and possibly enhance the athlete's performance and silver nanocrystals embedded in bandages to kill bacteria and prevent infection (Applications/Products). As an emerging technology, the future of Nanotechnology holds numerous potentials. For instance, Nanotechnology will make it possible to manufacture lighter, stronger and programmable materials that produce less waste than with conventional materials and that promise greater fuel efficiency in air and land transportation systems and space vehicles. It will engender fabrications of nanoscale electronic, magnetic and mechanical devices and systems with unprecedented levels of information processing as well as photochemical and biological sensors for protection; healthcare, environmental clean up, world security and defense and new photoelectric materials that will enable the production of cost efficient solar energy panels.

In this study, the techniques and tools involved in Nanotechnology, the underlying physical processes and factors that influence the properties of nanomaterials, the various applications and implications and the inherent challenges of nanotechnology are discussed.

\section{THE EMERGENCE OF NANOTECHNOLOGY}

The concept of nanotechnology originated with an American Nobelist and Physicist Richard P. Feynman. In a lecture to the American Society on 29 December 1959 entitled, There's Plenty of Room at the Bottom: an Invitation to Enter a New Field of Physics he presented his audience with a vision of what could be done with

Corresponding Author: J.C. Osuwa, Department of Physics, Micheal Okpara University of Agriculture, P.M.B. 7267, Umudike, Abia State, Nigeria 
extreme miniaturization. He began his lecture by calculating that the entire content of Encyclopedia Britannica could be reduced to fit on the head of a pin. $\mathrm{He}$ was also intrigued by biology and pointed out that cells are very tinny but they are very active, they manufacture various substances they walk around they wiggle and do all kinds of marvelous things all on a very small scale. Feynman believed the creation of nanoscale devices was possible within the boundaries set by the laws, knowledge, tools and methodologies used by physicists, chemists and biologists (Feynman, 1960).

Though Feynman did not coin the term nanotechnology, it was being promoted as a key component of future technology in the late 1970's. The term nanotechnology was first used in 1974 by Japanese scientist Norio Taniguchi in a study titled, On the Basic Concept of Nanotechnology (Taniguchi, 1974). An American scientist K. Eric Drexler starting with a 1981 study in the proceedings of the National Academy of Sciences and following with two books, Engines of Creation (1986) and Nanosystems (1992) became one of the foremost advocates of Nanotechnology (Drexler, 1986, 1992).

The prefix Nano, a Greek word for Dwarf, aptly describes the level of operation of Nanotechnology which is currently a highly interdisciplinary area of research interest involving physics, chemistry, biology and the full range of the engineering disciplines. It encompasses nanoscience which is concerned with the basic understanding of physical, chemical and biological properties of materials on atomic and near atomic scales and nanotechnology which employs the controlled manipulation of these properties to create materials and functional systems with unique capabilities.

\section{TOOLS AND TECHNIQUES IN NANOTECHNOLOGY}

The techniques currently adopted in the making of structures and devices at the nanoscale involve two approaches namely the top-down approach of miniaturizing current technologies and the bottom-up approach of building ever more complex molecular devices atom by atom. In the top-down process, technologists start with a bulk material and carve from it a smaller structure (Kubik et al., 2005). This approach is good for producing structures with long-range order and for making macroscopic connections. The top-down approach uses advanced lithographic techniques such as optical lithography and electron beam lithography to create structures as small as 100 and $20 \mathrm{~nm}$, respectively and efforts are being made to create structures smaller than the current computer chips. A key advantage of the top-down approach as developed in the fabrication of integrated circuits is that the parts are both patterned and built in place so that no assembly step is needed. This is seen in the production of computer chips in which the thin films of materials known as mask are deposited on a silicon wafer and the unwanted portions are etched away. These smaller and faster chips will enable computers to be smaller and perform many more functions more quickly.

In the second approach which is the bottom-up approach, technologists employ the method of self assembly. In this case, nanofabrication involves the application of chemical or physical forces operating at the nanoscale to assemble basic units into larger structures. Inspiration for bottom-up approaches comes from biological systems where nature has harnessed chemical forces to create essentially all structures needed in life and researchers are immersed in replicating nature's ability to produce small clusters of specific atoms which can self assemble into more-elaborate structures (Levins and Schafmeister, 2006). A number of bottom-up approaches have been developed for producing nanoparticles ranging from condensation of atomic vapors on surfaces to coalescence of atoms in liquids for example. Liquid-phase on inverse micelles (globules of lipid molecules floating in a non-aqueous solution in which their polar or hydrophilic ends point inwards to form a hollow core) has been developed to produce size-selected nanoparticles of magnetic and other materials. Another example of self-assembly of intricate structure is the formation of quantum dots (e.g., Indium Gallium Arsenide dots) and carbon nanotubes under the right set of chemical and temperature conditions.

Scientists achieved most of the research in nanoscience when tools became available, first to probe and later to manipulate and control at the nanoscale. These tools include the Transmission Electron Microscope (TEM), the Atomic Force Microscope (AFM) and the Scanning Tunneling Microscope (STM). The various microscopes function as follows.

TEM uses a high-energy electron beam to probe materials with a sample thickness of $<100 \mathrm{~nm}$. The electron beam is directed onto the object to be magnified. Some of the electrons are absorbed by or bounced off the object while others pass through the object and form a magnified image of the material. A photographic plate, fluorescent screen or digital camera placed behind the material records the magnified image. TEMs can magnify an object up to 30 million times in contrast to conventional optical microscope with only up to 1000 time magnification. They are suitable for imaging objects with dimensions of $<100 \mathrm{~nm}$ and they yield information on the size, 


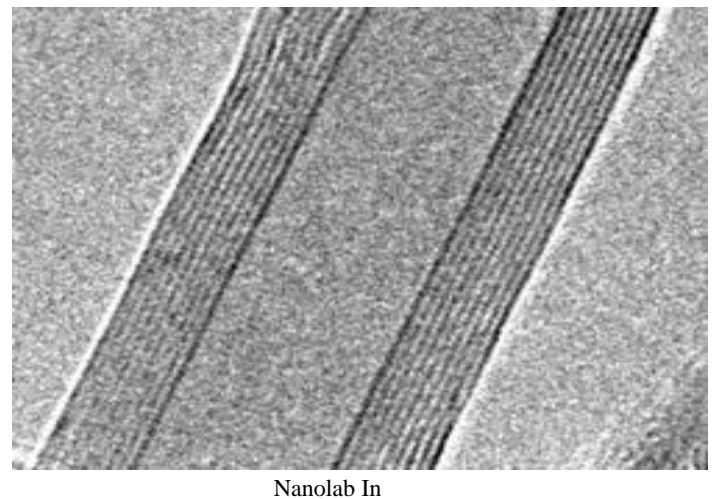

Fig. 1: TEM Image of a carbon nanotube

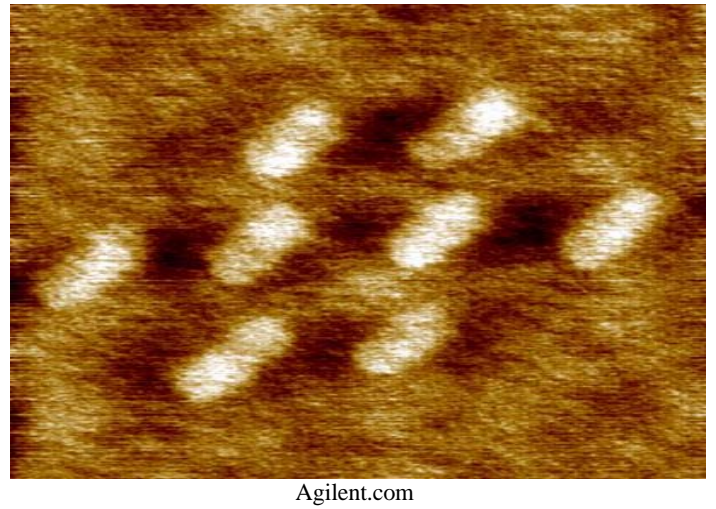

Fig. 2: AFM image of silicon semiconductor material

composition and crystal structures of nanomaterials. A TEM image of a carbon nanotube is shown in Fig. 1. The Atomic Force microscope uses a tinny silicon tip usually $<100 \mathrm{~nm}$ in diameter as a probe to create image of a sample material. As the silicon probe moves along the surface of the sample, electrons of the atoms in the sample repel the electrons in the probe. The AFM adjusts the height of the probe to keep the force on the sample constant. A sensing mechanism records the up-and-down movements of the probe and feeds the data into a computer which creates a three dimensional image of the surface of the sample. Thus, the exact surface topography is recorded with precise height information depicting the image of individual atoms in the surface. Figure 2 shows an image of a silicon semiconductor material obtained with AFM.

The Scanning Tunneling microscope also uses a tinny probe, the tip of which can be as small as a single atom to scan an object. An STM takes advantage of the wavelike property of electron called tunneling which allows electrons emitted from the probe of the microscope to penetrate or tunnel into the surface of the object being examined. The rate at which the electrons tunnel from the probe to the surface is related to the distance between the

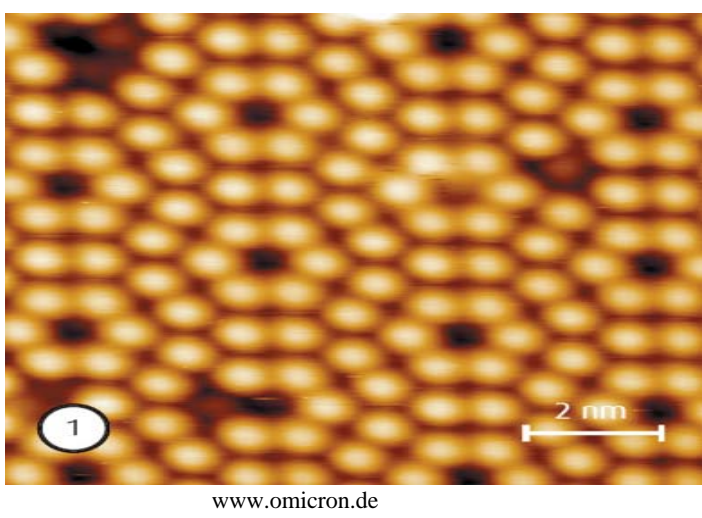

Fig. 3: STM image of silicon surface

probe and the surface. These moving electrons generate tinny electric current that the STM measures. The STM constantly adjusts the height of the probe to keep the current constant. By tracking how the height of the probe changes as the probe moves over the surface, scientists can get a detailed map of the surface. The map can be so detailed that individual atoms on the surface are visible. An STM image of a silicon surface is shown in Fig. 3.

\section{PROPERTIES AND EFFECTS OF MATERIALS AND DEVICES AT NANOSCALE}

There are two principal reasons for qualitative differences in material behavior at the nanoscale. First is the quantum effects which come into play at very small dimensions and lead to new physical and chemical properties of the material. Quantum effects arise in the nanoscale regime because the overall dimensions of objects are comparable to the characteristic wavelengths for fundamental excitations in materials. Such excitations include electrons, photons, phonons, magnons, etc. These excitations carry the quanta of energy through materials and thus determine the dynamics of their propagation and transformation from one form to another. Essentially, when the size of structures is comparable to the quanta themselves, it influences how these excitations move through and interact in the material. Thus, small structures may limit flow, create wave interference effects and bring into play quantum mechanical selection rules not apparent at large dimension. The second defining feature exhibited by materials at the nanoscale is the very large surface to volume ratio of these structures. This means that no atom is far from a surface or interface and the behavior of atoms at these higher-energy sites have significant influence on the properties of the materials. For example, the reactivity of a metal catalyst particle generally increases appreciably as the size is reduced 
macroscopic gold is chemically inert whereas at nanoscale gold becomes extremely reactive and catalytic and even melts at a lower temperature. Thus, at nanoscale dimensions, material properties depend and change with size as well as composition and structure (Regan et al., 2005). In solid state electronics, quantum mechanical properties for confinement of electrons in one dimension have long been exploited.

Semiconductor devices are grown with thin layers of differing composition so that electrons or holes can be confined in specific regions of the structure (known as quantum wells). Large energy band gaps associated with thin layers can serve as barriers that restrict the flow of charges to such conditions that cause them to tunnel through the barriers-this forms the basis of resonant tunneling diodes. Modern telecommunication is based on semiconductor lasers that exploit the unique properties of quantum wells to achieve specific wavelengths and high efficiency.

The propagation of photons is drastically altered when the size and periodicity of the transient structure approach the wavelength of visible light. Photons propagating through a periodically varying dielectric constant such as semiconductor posts surrounded by air are subject to quantum mechanical rules that define and limit their propagation depending on their energy (wavelength). This is analogous to the quantum mechanical rules that define the motion of electrons through crystals, giving band gaps for semiconductors.

Nanoscale materials also have size-dependent magnetic behavior, mechanical properties and chemical reactivity at very small sizes of a few nanometers, magnetic clusters have a single magnetic domain and the strongly coupled magnetic spins of individual atoms produce a particle with a single giant spin. For example, the giant spin of a ferromagnetic iron particle rotates freely at room temperature for diameters below $16 \mathrm{~nm}$, an effect termed superparamagnetism. Mechanical properties of nanostructured materials can reach exceptional strength. For example, the introduction of 2 nm aluminum oxide precipitates into thin films of pure nickel results in yield strengths increasing from $0.15-5$ gigapascals which is more than twice that for hard bearing steel.

Due to predominance of surfaces, up to half of all the atoms in the nanoparticles are surface atoms and properties such as electrical transport are no longer determined by solid-state bulk phenomena. Also, the atoms in nanostructures have a higher average energy than atoms in larger structures because of the large proportion of surface atoms. For example, catalytic materials such as gold particles have a greater chemical activity per atom of exposed surface as the catalyst is reduced in size at the nanoscale. At these dimensions, atomic and chemical forces dominate while macroscopic effects-such as convention, turbulence and momentum (inertial forces) are of little consequence (Drexler, 1992).

\section{APPLICATIONS OF NANOTECHNOLOGY}

Increased relative surface area and quantum effects as discussed in the preceding section change or enhance the properties of nanomaterials and render them versatile for a wide range of applications as follows. They are used in cost effective and efficient solar cell devices in which energy transfers from nano-thin layers of quantum wells to nanocrystals above them cause the nanocrystals to emit visible light. Nanomaterials also make excellent filters for trapping heavy metals and other pollutants from industrial wastewater and also in pollution control systems (Appliications/products).

Nanoelectrononics has increased the capabilities of electronic devices along with significant reduction in weight. High performance electronic devices have been achieved through increased density, speed and reduction in power consumption. These quantum electronic devices are designed and synthesized using batch processes of self-organization and self-alignment. The emergence of nanotechnology has also made important impact in the areas of production of high performance systems in telecommunications for signal processors and electronics in analogue-digital converters in detectors, resonant tunneling devices in logic and memory circuits, improved display screens on electronic devices, increased density of memory chips and reduction in the size of transistors used in integrated circuits. Also, electrodes made from nanowires would enable flat panel displays to be flexible and thinner than the current ones, production of nanowires for building transistors without $\mathrm{p}-\mathrm{n}$ junctions, nanosize magnetic rings for making Magneto resistive Random Access Memory (MRAM) and the production of gold particles with organic molecules to create a transistor known as NOMFET (Nanoparticle Organic Memory Field-Effect Transistor).

In medicine, nanodevices have been produced for diagnosis, treatment and prevention of diseases and for traumatic surgery, pains relief and in overall improvement of human health. In nano-pharmacology, biologically active compounds or molecules are linked with specially fabricated nanomaterials. These are then used as probes and drugs to identify and target various abnormal cells in diseased conditions. Smart drugs which are new advanced nanosized therapeutic drugs have been developed and administered with high efficiency and 
minimal side effects. These drugs are delivered at the precise location in the body and the drug doses are released on a predetermined schedule for optimal treatment. New types of nanosensors with ultrahigh sensitivity and specificity can detect cancerous tumors when they consist of only a few cells. Medical Nanorobots when injected into the blood stream would travel freely throughout the body, identify abnormal cells in the body and supply the drugs carried by them selectively on coming in contact with the diseased or abnormal cells. Nanotweezers are surgical toolsselectively controlled multicoated carbon nanotubes-used to grab any single biological molecule within the cell. It helps doctors perform surgery on an individual human cell (Ghalanbor et al., 2005; Kubik et al., 2005).

\section{CHALLENGES AND IMPLICATIONS OF NANOTECHNOLOGY}

As with any transformative technology, there are serious concerns about what effects nanotechnology with its far ranging potentials and applications will have on the society as a whole, on human life and environment, on the food chain, economy, jobs, etc. There are fears that new developments in nanotechnology could result in untraceable weapons of mass destruction, networked cameras for use by government and weapons development fast enough to destabilize arms race.

Another fear is the effect of these nanomaterials on human health and environment. Silver nanoparticles used in socks to reduce foot odor are being released in the wash. These nanoparticles destroy beneficial bacteria for breaking down organic matter in waste treatment plants (Murray et al., 2004). Researchers at UCLA's School of Public Health found that lab mice consuming nanotitanium oxide showed DNA and chromosome damage which can be linked to man's major diseases like cancer, aging, heart and neurological diseases. According to Anthony Seaton's contribution on Carbon nanotubes, he said, we know that some of them probably have the potential to cause Mesothelioma so those sorts of materials need to be handled very carefully. As a result, some organizations have called for stricter Regulations for Nanotechnology. They include: Institute for Food and Agriculture, Department of Toxic Substances Control, California and Center for Responsible Nanotechnology. In addition, Nanotechnology will create many new jobs as well as make other jobs obsolete since it is aimed at manufacturing less bulky, cheaper and more efficient products. For example, jobs manufacturing conventional televisions will be made obsolete as nanotechnology based Field-Emission and Liquid Crystal Display televisions will be preferred.

\section{CONCLUSION}

A treatise of Nanotechnology at its conceptual, developmental and practical stages has been presented. The underlying physical processes that characterize the unique and novel properties of nanomaterials have been discussed.

The immense applications in the areas of production of high performance and efficient electronic and optoelectronic devices, in information and communication technology, in medicine and other aspects of human needs have also been provided.

Enormous challenges and implications in efforts to harness and replicate natural processes at the nanoscale are evident.

\section{REFERENCES}

Drexler, K.E., 1986. Engines of Creation: The Coming Era of Nanotechnology. Anchor/Double Day Books, New York.

Drexler, K.E., 1992. Nanosystems: Molecular Machinery, Manufacturing and Computation. John Wiley and Sons, New York, pp: 556

Feynman, R.P., 1960. There's plenty of room at the bottom. Caltech's Engineering and Science. Lect. to the American Physical Society. December 1959. http://www.zyvex.com/nanotech/feynman.html.

Ghalanbor, Z., S.A. Marashi and B. Ranjbar, 2005. Nanotechnology helps medicine: Nanoscale swimmers and their future applications. Med. Hypothesis, 65: 198-199.

Kubik, T., K. Bogunia-Kubik and M. Sugisaka, 2005. Nanotechnology on duty in medical applications. Curr. Pharm. Biotechnol., 6: 17-33.

Levins, C.G. and C.E. Schafmeister, 2006. The synthesis of curved and linear structures from a minimal set of monomers. ChemInform, Vol. 37. 10.1002/chin.20060 5222 .

Murray, R.G.E., T.J. Beverage and S.F. Koval, 2004. Advances in Bacterial Paracrystalline Surface Layers. Plenum Publishers, New York.

Regan, B.C., S. Aloni, K. Jensen, R.O. Ritchie and A. Zettl, 2005. Nanocrystal-powered nanomotor. Nanoletters, 5: $1730-1733$.

Taniguchi, N., 1974. On the basic concept of nano-technology. Proceedings of the International Conference on Production Engineering, London Part II British Society of Precision Engineering. 